\title{
K-conjugated Dibenzoazahexacenes
}

Gabriella Antonicelli, ${ }^{\mathrm{a}}$ Cristian Gozalvez, ${ }^{\mathrm{a}}$ Ainhoa Atxabal, ${ }^{\mathrm{b}}$ Manuel Melle-Franco, ${ }^{\mathrm{c} *}$ Luis E. Hueso ${ }^{\mathrm{b}, \mathrm{d} *}$ and Aurelio Mateo-Alonso ${ }^{\mathrm{a}, \mathrm{d} *}$

${ }^{a}$ POLYMAT, University of the Basque Country UPV/EHU. Avenida de Tolosa 72, E-20018 Donostia-San Sebastián, Spain.

${ }^{b}$ CIC Nanogune. Avenida de Tolosa 76, E-20018 Donostia-San Sebastián, Spain.

${ }^{c}$ CICECO - Aveiro Institute of Materials, Department of Chemistry, University of Aveiro, 3810-193 Aveiro, Portugal.

${ }^{d}$ Ikerbasque, Basque Foundation for Science. Bilbao, Spain.

\section{Supporting Information}

Contents

page

1. Experimental details

S1

2. Synthesis and characterization of compounds 1 and 2

3. NMR spectra for compounds 1 and 2

S6

4. MALDI-TOF for compounds 1 and 2

5. Thermal behavior

S9

6. Cyclic voltammetry

7. Single crystal X-ray diffraction data compounds 1 and 2

8. Devices

9. AFM 


\section{Experimental details}

All commercially available reagents and solvents were used without further purification unless otherwise specified. Anhydrous THF and toluene were dried through a SPS solvent purification system. All reactions were carried out under inert atmosphere of nitrogen and oven-dried glassware, unless stated otherwise.

Column chromatography was carried out using Silica gel $60(40-60 \mu \mathrm{m})(230-400 \mathrm{mesh})$ from VWR. Thin layer chromatography (TLC) was performed to follow the reaction process by using sheets $(20 \times 20)$ of aluminum pre-coated with silica gel $60 \mathrm{~F}_{254}$ from Merck. UV-active compounds were detected with a UV-lamp from CAMAG at wavelength $\lambda=254$ or $366 \mathrm{~nm}$.

${ }^{1} \mathrm{H}-\mathrm{NMR}$ and ${ }^{13} \mathrm{C}-\mathrm{NMR}$ spectra were recorded on Bruker Avance 400 spectrometer at $298 \mathrm{~K}$ using partially deuterated solvents as internal references. Chemical shifts are reported in $(\delta)$ ppm and referred to TMS. Multiplicities are denoted as follows: $\mathrm{s}=$ singlet, $\mathrm{d}=\operatorname{doublet} \mathrm{t}=$ triplet, $\mathrm{m}=$ multiplet, $\mathrm{br}=$ broad. ${ }^{1} \mathrm{H}$ NMR data were reported in order: chemical shift, multiplicity (s, singlet; d, doublet; t, triplet; q, quartet; m, multiplet), coupling constants ( $\mathrm{J}, \mathrm{Hz}$ ), number of protons.

Matrix Assisted Laser Desorption Ionization (coupled to a Time-Of-Flight analyzer) experiments (MALDI-TOF) were recorded on Bruker REFLEX spectrometer in Polymat by Dr. Antonio Veloso.

Absorption and emission spectra were recorded on a Perkin-Elmer Lambda 950 spectrometer, and a LS55 Perkin-Elmer Fluorescence spectrometer, respectively. Anhydrous 1,2dichlorobenzene from Sigma-Aldrich was used as purchased.

TGA Q500 TA Instruments, was used to perform the thermogravimetric analysis (TGA) using a $10^{\circ} \mathrm{C} /$ min heating rate under a nitrogen flow, which was changed to oxygen from $800^{\circ} \mathrm{C}$.

Electrochemical measurements were carried out on a Princeton Applied Research Parstat 2273 in a 3-electrode single compartment cell with Pt disc working electrode $(\varnothing=0.5 \mathrm{~mm})$, a platinum wire counter electrode $(\varnothing=0.5 \mathrm{~mm})$ and a silver wire pseudoreference electrode. The cell and the electrodes were custom made. The reduction potentials were referred to SCE using ferrocene $(\mathrm{Fc})$ as internal reference $\left(\mathrm{E}_{1 / 2} \mathrm{FcSCE}=+0.48 \mathrm{~V}\right)$ after the measurements. 
X-ray diffraction experiments were performed by the X-ray diffraction unit of General Services SG-Iker (UPV/EHU) by Dr. Leire San Felices. Intensity data were collected on an Agilent Technologies Super-Nova diffractometer, wich was equipped with monochromated $\mathrm{Cu} k \alpha$ radiation $(\lambda=1.54184 \AA)$ and Atlas CCD detector. Measurement was carried out at 150.00(10) K with the help of an Oxford Cryostream 700 PLUS temperature device. Data frames were processed (united cell determination, analytical absorption correction with face indexing, intensity data integration and correction for Lorentz and polarization effects) using the Crysalis software package. The structure was solved using Olex 2 and refined by full-matrix least-squares with SHELXL-97. Final geometrical calculations were carried out with Mercury and PLATON as integrated in WinGX.

The electrical characterizations of the devices were measured with a Keithley 4200 semiconductor analyzer system which connected to Lakeshore probe-station in vacuum at room temperature in the dark.

The mobility of each electrode was calculated in the saturation regime using Equation 1:

$$
I_{D S, s a t}=\frac{C i W}{2 L} \mu_{F E}\left(V_{G S}-V_{T}\right) \quad \text { Equation 1 }
$$

Where $\mu_{\mathrm{FE}}$ is the field-effect mobility, $C i$ is the capacitance per unit area of the dielectric layer, $L$ is the channel length, $W$ is the channel width, $V_{\mathrm{T}}$ is the threshold voltage and $V_{\mathrm{GS}}$ is the gatesource bias.

AFM images were taken by a commercial Agilent Atomic Force Microscope (AFM) with commercial Bruker NCHV tips in tapping mode. The scanned area of the samples was $5 \mu \mathrm{m} \times$ $5 \mu \mathrm{m}$ with 512 points/lines resolution. 


\section{Synthesis and characterization of compounds 1 and 2}

Preparation starting materials. 4-bis((triisopropylsilyl)ethynyl)anthracene-2,3-diamine ${ }^{[1]} 1,4-$ bis((triisopropylsilyl)ethynyl)phenazine-2,3-diamine ${ }^{[2]}$ and pyrene diketone ${ }^{[3]}$ were synthesized according to reported procedures.

General procedure (GP). In a schlenk flask, 1 eq. of pyrene diketone and 1.1 eq of diamine were dissolved in a mixture of acetic acid and chloroform 1:1. The mixture was heated to reflux and stirred overnight. After this time, reaction was cooled to room temperature and quenched with water. The organic phases were washed with sat. $\mathrm{NaHCO}_{3}(50 \mathrm{~mL})$ and brine $(50 \mathrm{~mL})$ and dried over $\mathrm{Na}_{2} \mathrm{SO}_{4}$. The solvent was removed in vacuo and the crude product was purified by column chromatography.

\section{Compound 1:}

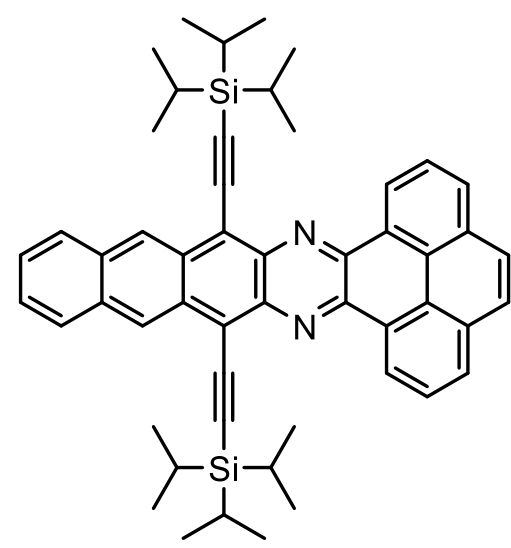

The general procedure was followed using $3(183.6 \mathrm{mg}, 0.7907 \mathrm{mmol})$ and $4(409 \mathrm{mg}, 0.719$ $\mathrm{mmol})$ in a mixture of acetic acid $(15 \mathrm{~mL})$ and chloroform $(15 \mathrm{~mL})$. Column chromatography (Hexane: $\mathrm{CHCl}_{3}$ 9:1) afforded compound 1 as purple solid (205 mg, 0.268mmol, 37\%). ${ }^{1} \mathrm{H} \mathrm{NMR}$ (400 MHz, Chloroform- $d$ ): $\delta 9.72(\mathrm{~d}, J=7.6 \mathrm{~Hz}, 2 \mathrm{H}), 9.50(\mathrm{~s}, 2 \mathrm{H}), 8.26(\mathrm{~d}, J=7.7 \mathrm{~Hz}, 2 \mathrm{H})$, $8.08(\mathrm{dt}, J=6.5,3.2 \mathrm{~Hz}, 2 \mathrm{H}), 8.04-7.96(\mathrm{~m}, 4 \mathrm{H}), 7.55(\mathrm{dt}, J=6.7,3.3 \mathrm{~Hz}, 2 \mathrm{H}), 1.45(\mathrm{~d}, J=$ $4.4 \mathrm{~Hz}, 43 \mathrm{H}) .{ }^{13} \mathrm{C} \mathrm{NMR}\left(101 \mathrm{MHz}, \mathrm{CDCl}_{3}\right): \delta 145.55,140.64,133.23,132.75,132.03,130.74$, 130.26, 129.16, 127.63, 127.40, 127.30, 127.21, 127.09, 125.87, 120.93, 108.46, 104.18, 19.54, 12.31. MS (MALDI-TOF) (m/z): calculated for $[\mathrm{M}]^{+} \mathrm{C}_{52} \mathrm{H}_{56} \mathrm{~N}_{2} \mathrm{Si}: 764.398$; found: 764.558 . 


\section{Compound 2:}

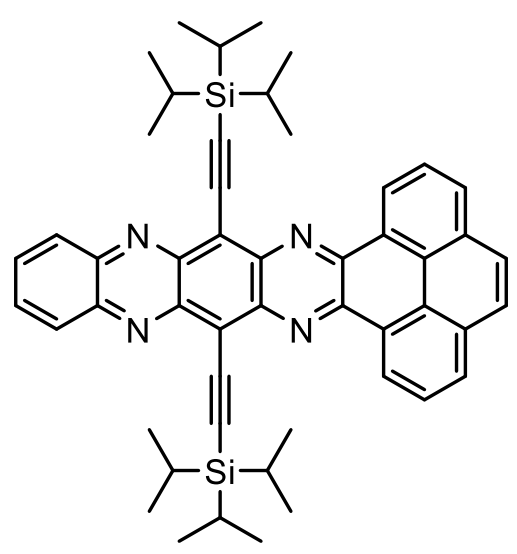

The general procedure was followed using $3(75 \mathrm{mg}, 0.1313 \mathrm{mmol})$ and $5(33.5 \mathrm{mg}$, $0.1415 \mathrm{mmol})$ in a mixture of acetic acid $(5 \mathrm{~mL})$ and chloroform $(5 \mathrm{~mL})$. Column chromatography (Hexane: $\mathrm{CHCl}_{3}$ 9:1) afforded compound 2 as purple solid (51 $\mathrm{mg}, 0.065 \mathrm{mmol}$, 51\%). ${ }^{1} \mathrm{H}$ NMR (400 MHz, Chloroform- $d$ ): $\delta 9.78(\mathrm{dd}, J=7.8,1.2 \mathrm{~Hz}, 2 \mathrm{H}), 8.36-8.28$ (m, $4 \mathrm{H}), 8.09-8.03(\mathrm{~m}, 4 \mathrm{H}), 7.91(\mathrm{dd}, J=6.9,3.4 \mathrm{~Hz}, 2 \mathrm{H}), 1.47(\mathrm{~d}, J=2.9 \mathrm{~Hz}, 42 \mathrm{H}) .{ }^{13} \mathrm{C} \mathrm{NMR}$ $\left(101 \mathrm{MHz}, \mathrm{CDCl}_{3}\right): \delta 145.90,144.78,142.91,142.28,131.52,131.47,130.76,130.27,129.31$, $127.07,126.95,126.74,125.80,122.14,110.52,102.59,18.87,11.72$. MS (MALDI-TOF) $(\mathrm{m} / \mathrm{z})$ : calculated for $[\mathrm{M}+\mathrm{H}]^{+}\left(\mathrm{C}_{50} \mathrm{H}_{54} \mathrm{~N}_{4} \mathrm{Si}_{2}\right)=767.397$, found $=767.520$. 
3. NMR spectra for compounds 1 and 2

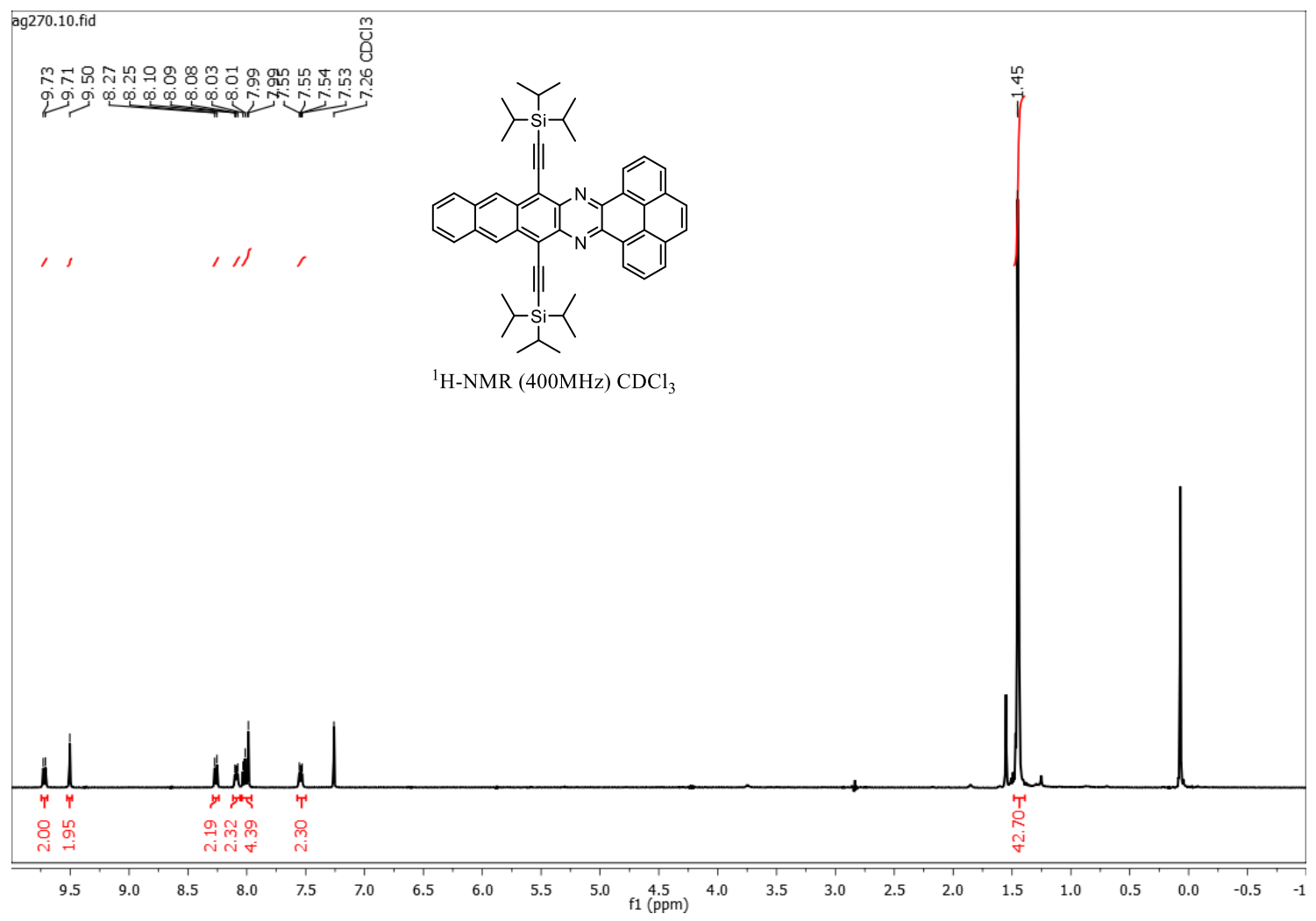

Figure S1: ${ }^{1} \mathrm{H}$ NMR-Spectrum of compound $\mathbf{1}$ in $\mathrm{CDCl}_{3}$

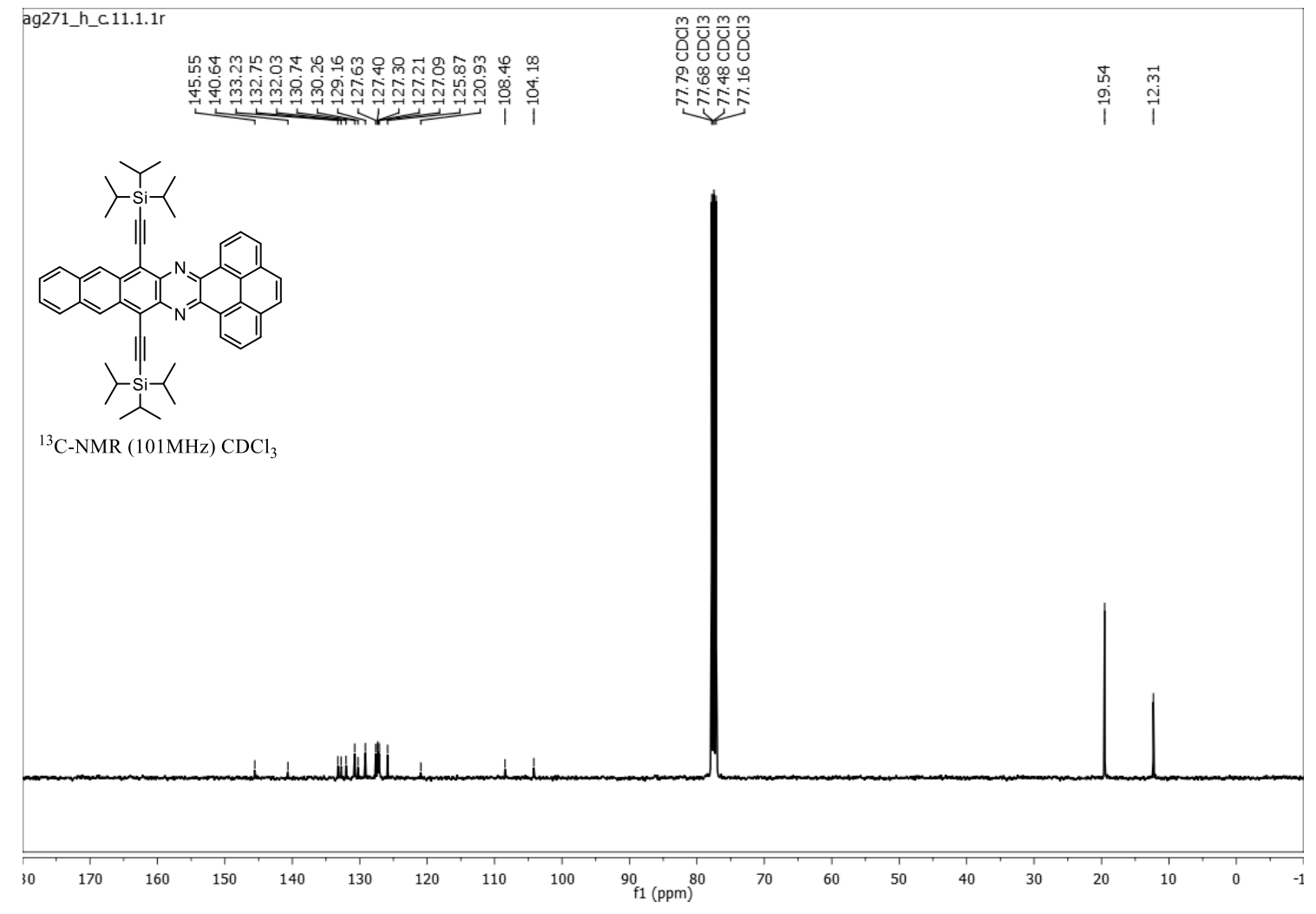

Figure S2: ${ }^{13} \mathrm{C}$ NMR-Spectrum of compound $\mathbf{1}$ in $\mathrm{CDCl}_{3}$

Sb 


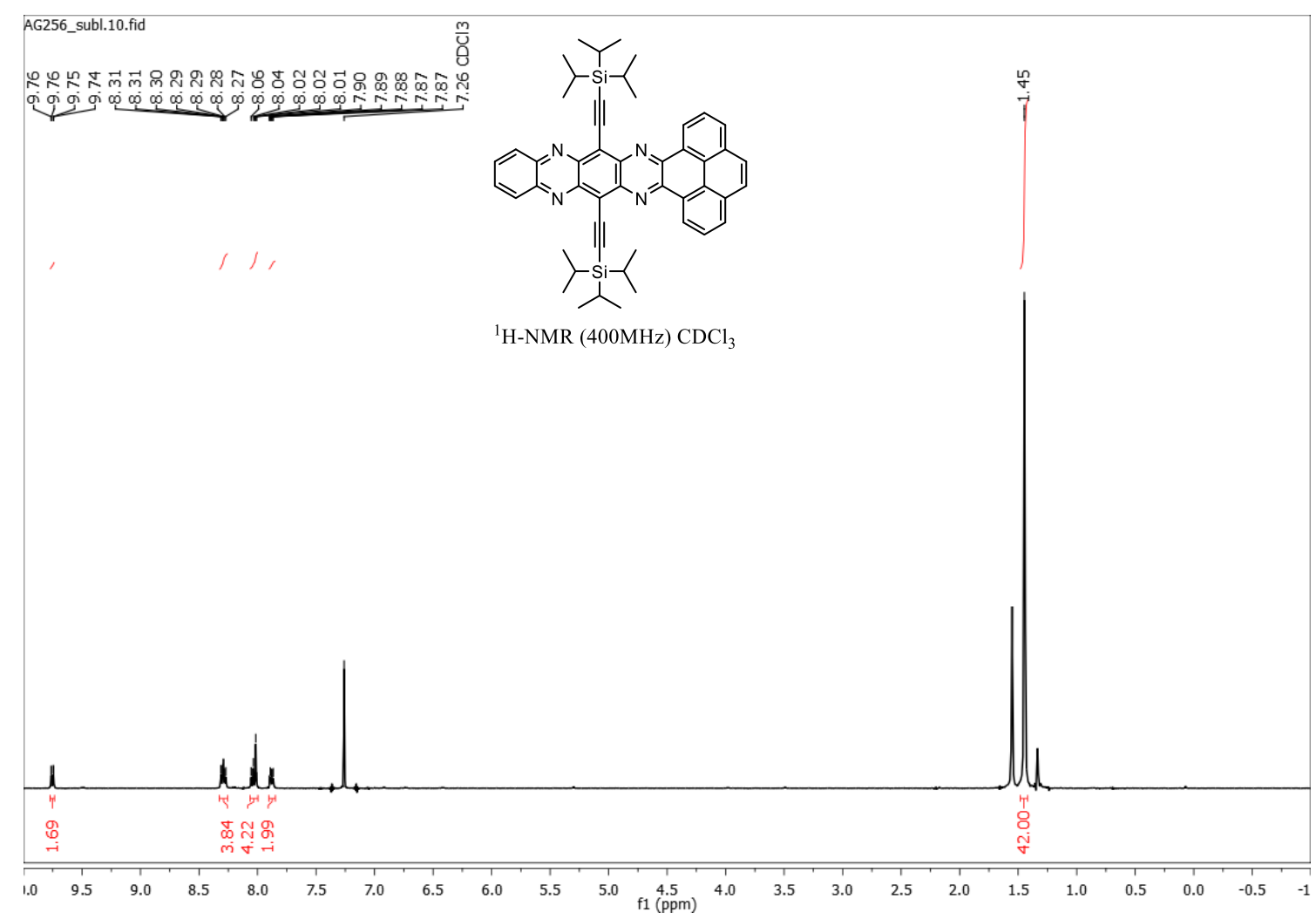

Figure S3: ${ }^{1} \mathrm{H}$ NMR-Spectrum of compound 2 in $\mathrm{CDCl}_{3}$

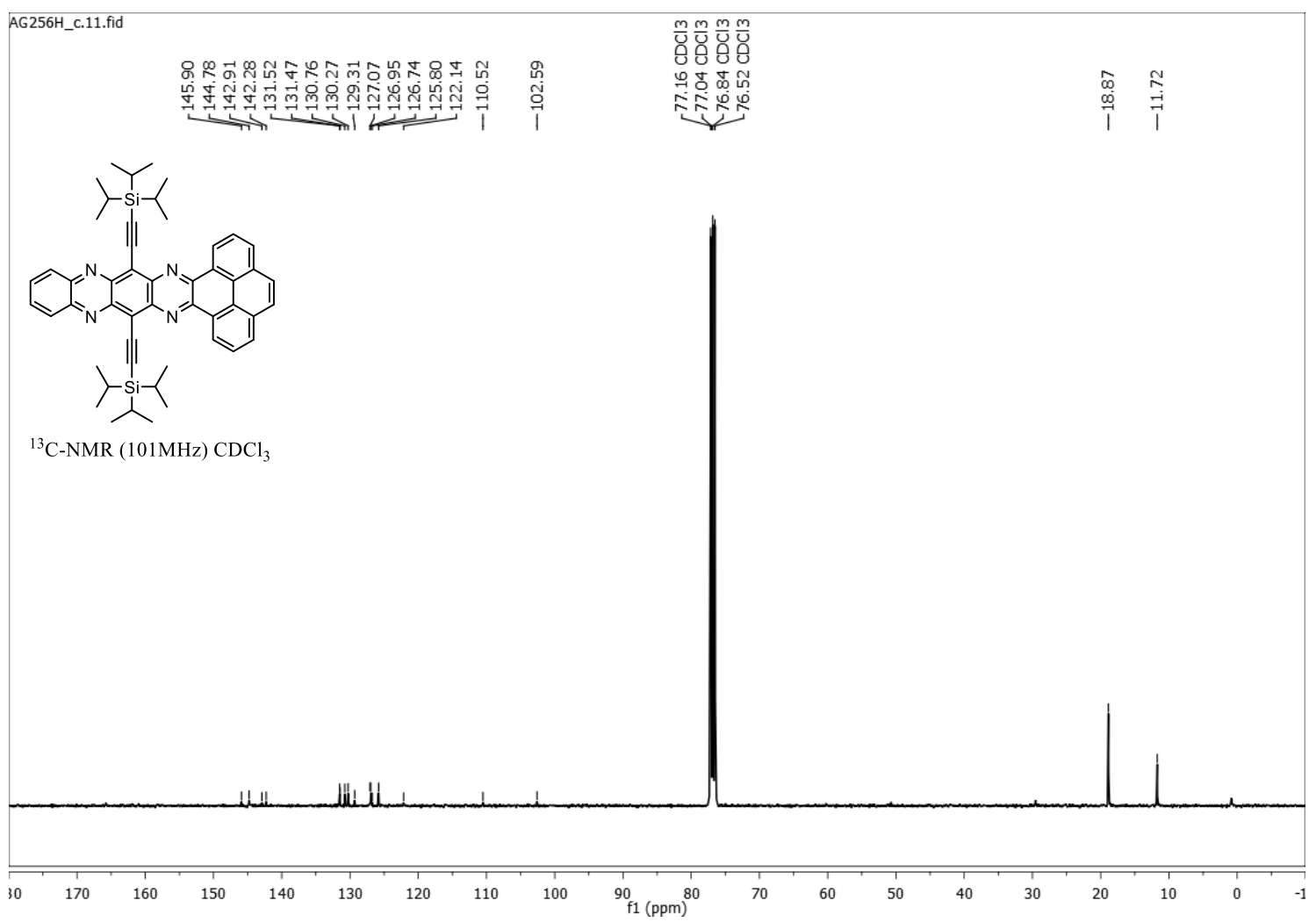

Figure S4: ${ }^{13} \mathrm{C}$ NMR-Spectrum of compound 2 in $\mathrm{CDCl}_{3}$ 


\section{MALDI-TOF for compounds 1 and 2}

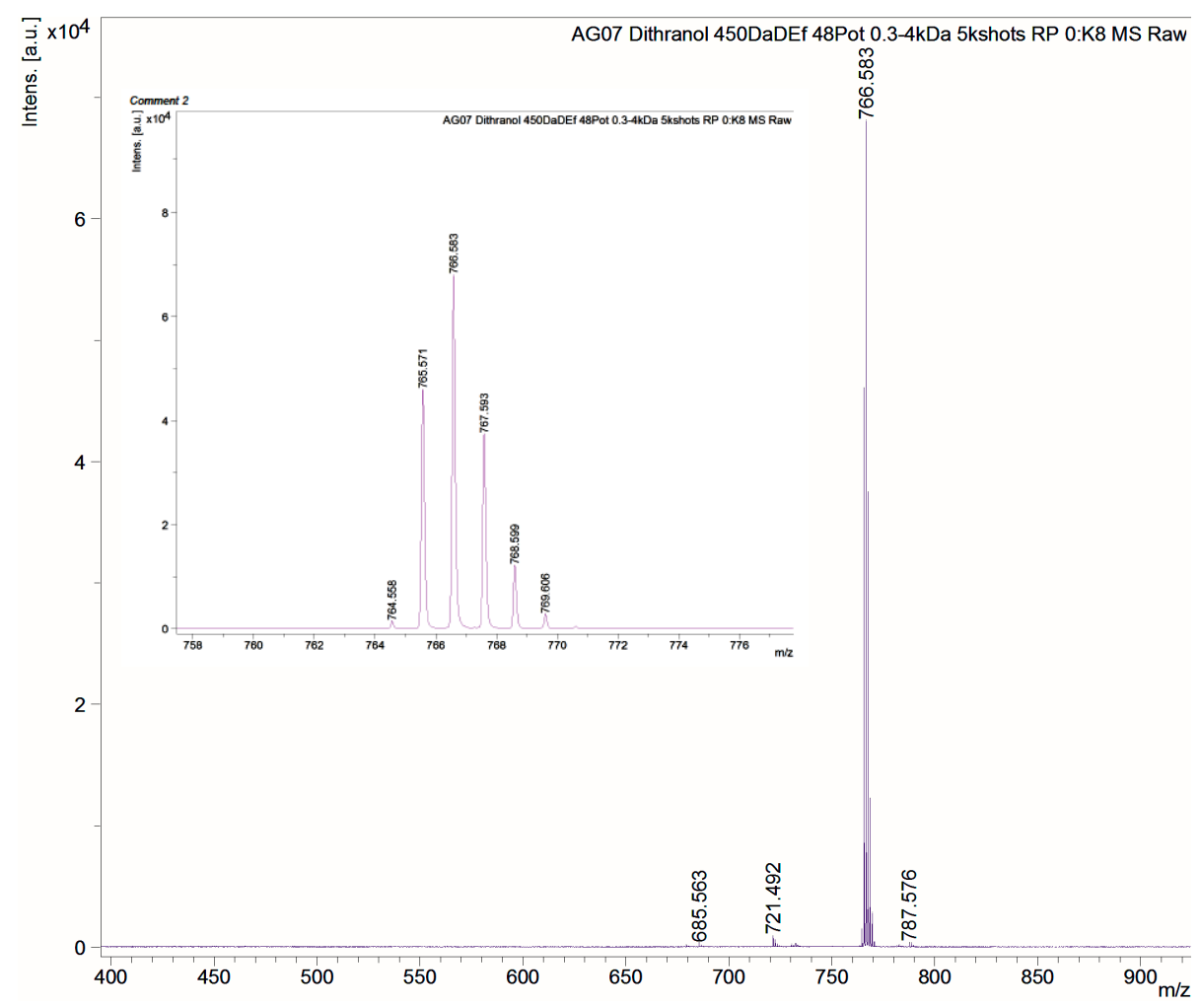

Figure S5: MALDI-TOF compound 1

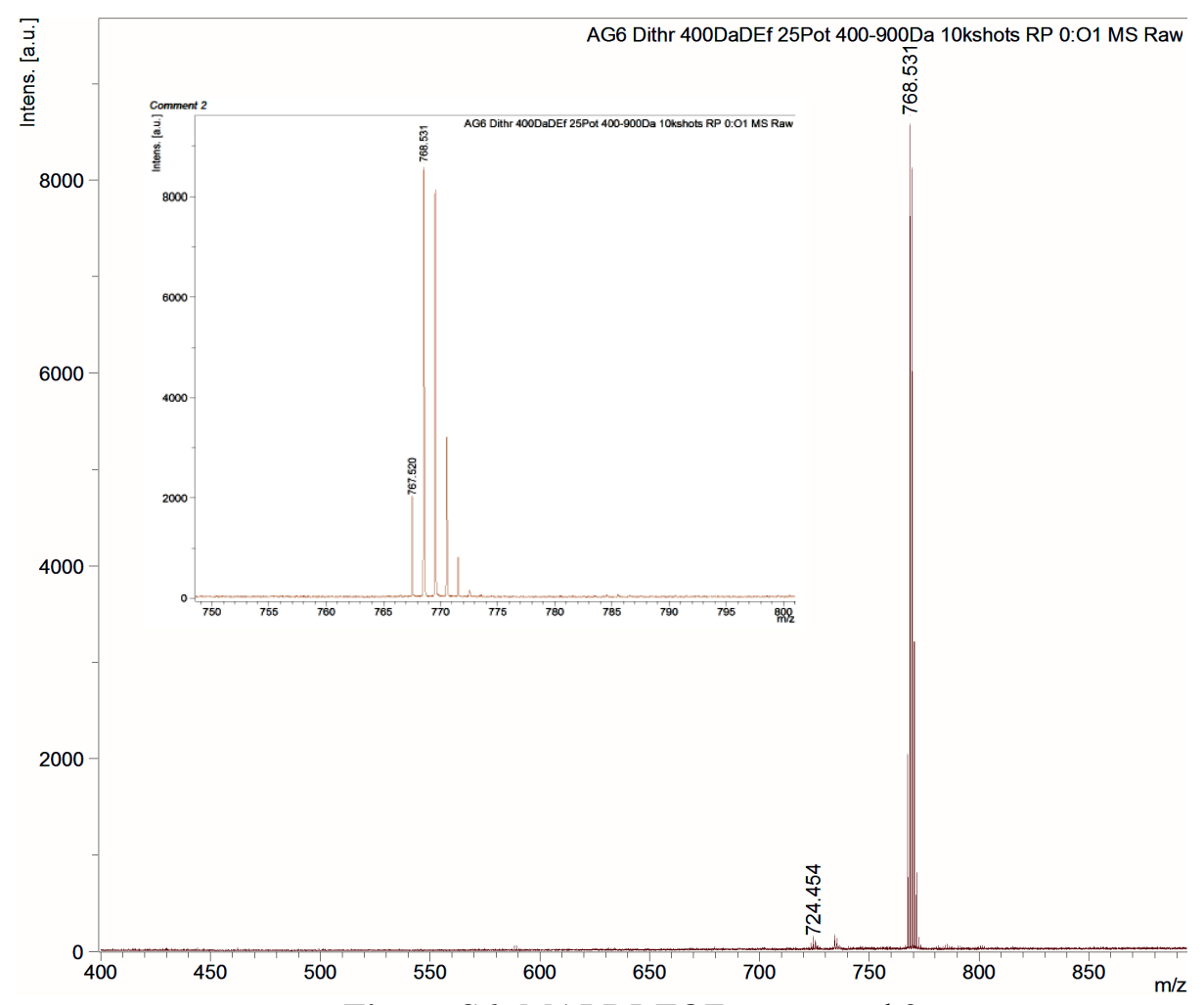

Figure S6: MALDI-TOF compound 2 


\section{Thermal behavior}

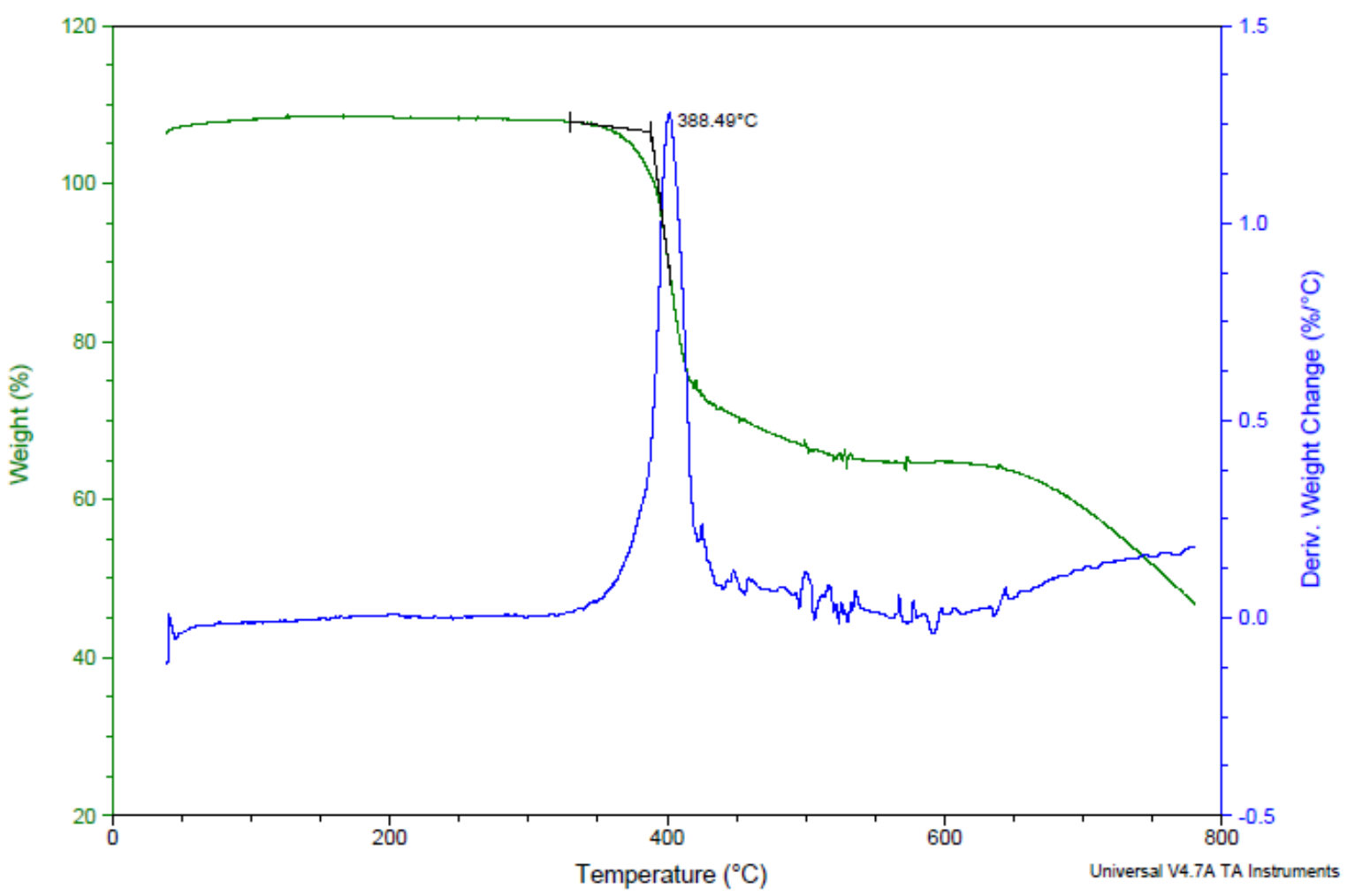

Figure S7: TGA compound 1

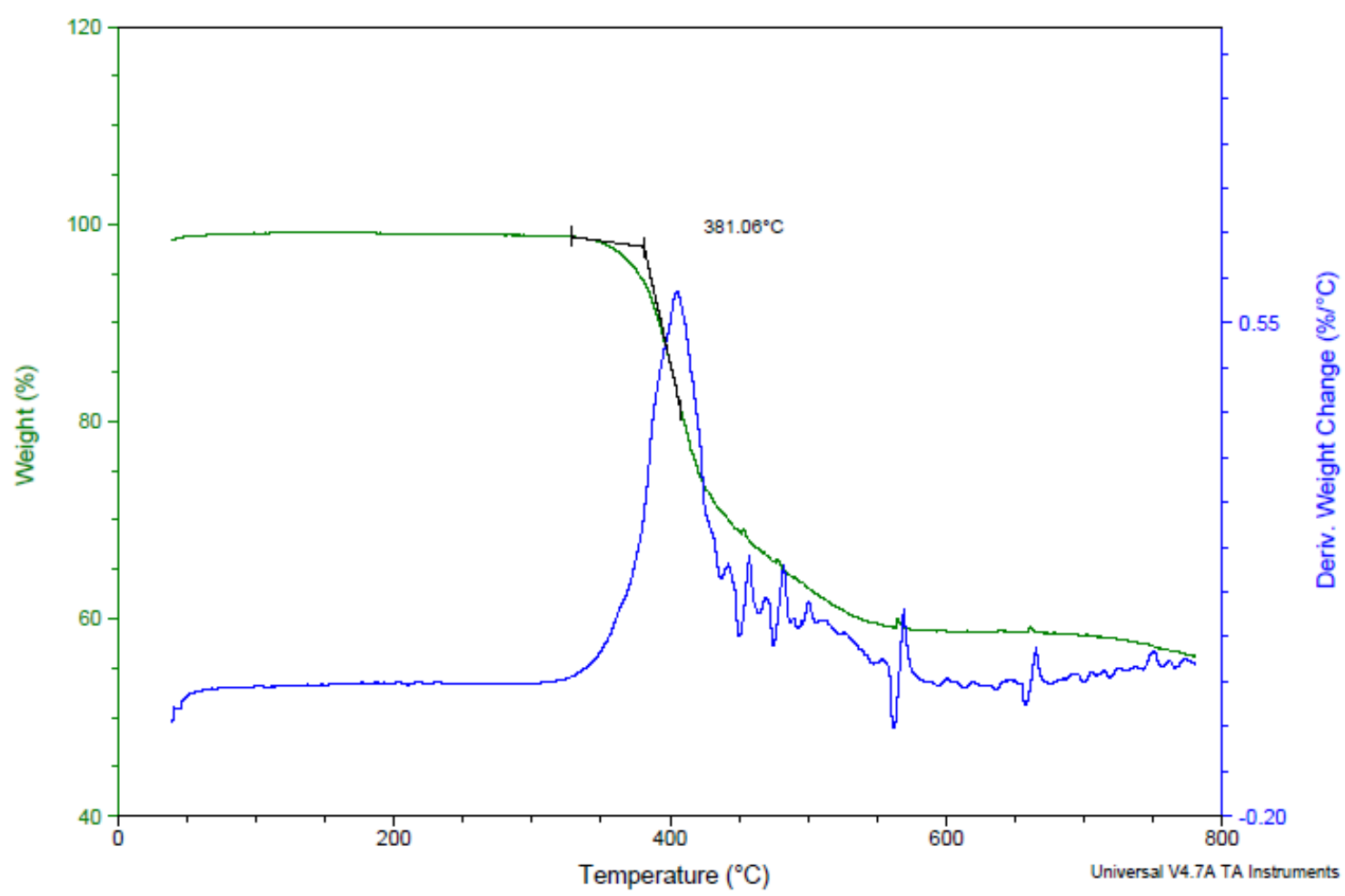

Figure S8: TGA compound 2 


\section{Cyclic Voltammetry}

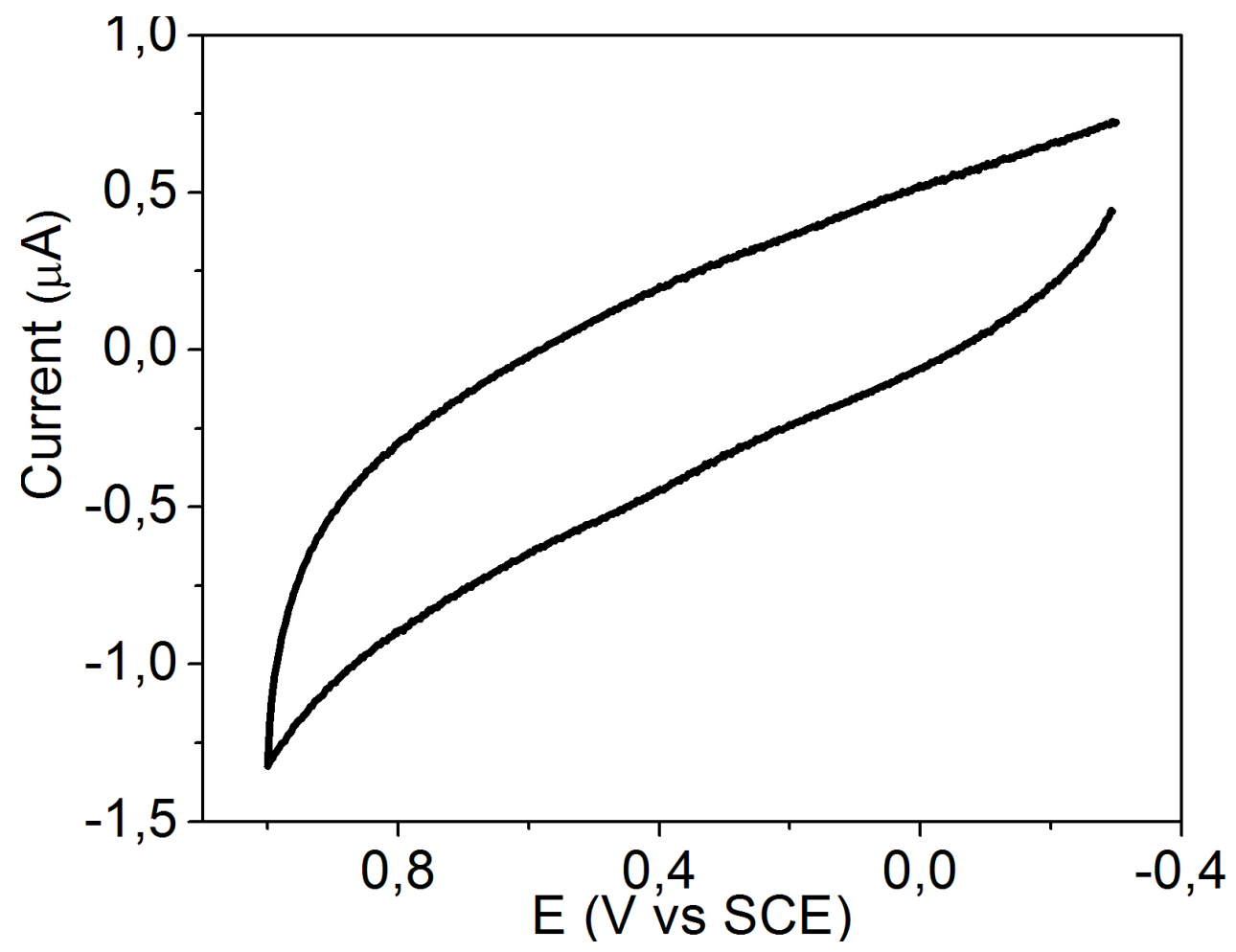

Figure S9: Cyclic voltammetry of $\mathbf{2}$ measured in ODCB for oxidation 


\section{X-Ray Diffraction data for compounds 1 and 2}

Datablock: a20160196_ag271

Bond precision: $\mathrm{C}-\mathrm{C}=0.0050 \mathrm{~A}$

Wavelength $=1.54184$

Cell:

$\mathrm{a}=7.8003(9)$

$\mathrm{b}=16.2905(9)$

$\mathrm{c}=17.4071(12)$

alpha $=79.797(5)$

beta $=87.838(7)$

gamma $=87.209(6)$

Temperature: $150 \mathrm{~K}$

$\begin{array}{lll} & \text { Calculated } & \text { Reported } \\ \text { Volume } & 2173.4(3) & 2173.4(3) \\ \text { Space group } & \mathrm{P}-1 & \mathrm{P}-1 \\ \text { Hall group } & -\mathrm{P} 1 & -\mathrm{P} 1 \\ \text { Moiety formula } & \text { C52 H56 N2 Si2 } & \text { C52 H56 N2 Si2 } \\ \text { Sum formula } & \text { C52 H56 N2 Si2 } & \text { C52 H56 N2 Si2 } \\ \text { Mr } & 765.17 & 765.16 \\ \text { Dx,g cm-3 } & 1.169 & 1.169 \\ \text { Z 2 2 Mu (mm-1) } & 1.012 & 1.012 \\ \text { F000 } & 820.0 & 820.0 \\ \text { F000, } & 822.91 & \\ \text { h,k,lmax } & 9,19,21 & 9,19,21 \\ \text { Nref } & 8102 & 8071 \\ \text { Tmin,Tmax } & 0.970,0.979 & 0.568,1.000 \\ \text { Tmin' } & 0.883 & \end{array}$

Correction method $=\#$ Reported $\mathrm{T}$

Limits: $\mathrm{Tmin}=0.568$

$\operatorname{Tmax}=1.000$

AbsCorr $=$ MULTI-SCAN

Data completeness $=0.996$

$\operatorname{Theta}(\max )=68.996$

$\mathrm{R}($ reflections $)=0.0729(5094)$

$\mathrm{wR} 2($ reflections $)=0.2101(8071)$

$\mathrm{S}=1.037 \quad \mathrm{Npar}=517$

Table S1. Crystal data and structure refinement for compound 1 
Datablock: a20160227_ag256a075

Bond precision: $\mathrm{C}-\mathrm{C}=0.0083 \mathrm{~A}$ Wavelength $=1.54184$

Cell:

$$
\begin{aligned}
& \mathrm{a}=7.7643(8) \\
& \text { alpha=80.174(9) }
\end{aligned}
$$$$
b=16.1450(16)
$$$$
\mathrm{c}=17.4579(19)
$$$$
\text { beta }=88.112(9)
$$$$
\text { gamma }=87.207(8)
$$

Temperature: $150 \mathrm{~K}$

\begin{tabular}{lll} 
& Calculated & Reported \\
Volume & $2153.1(4)$ & $2153.1(4)$ \\
Space group & $\mathrm{P}-1$ & $\mathrm{P}-1$ \\
Hall group & $-\mathrm{P} 1$ & $-\mathrm{P} 1$ \\
Moiety formula & C50 H54 N4 Si2 & \multicolumn{1}{c}{ C50 H54 N4 Si2 } \\
Sum formula & C50 H54 N4 Si2 & C50 H54 N4 Si2 \\
Mr & 767.15 & 767.15 \\
Dx,g cm-3 & 1.183 & 1.183 \\
Z & 2 & 2 \\
Mu (mm-1) & 1.037 & 1.037 \\
F000 & 820.0 & 820.0 \\
F000' & 822.94 & \\
h,k,lmax & $9,19,21$ & $9,19,21$ \\
Nref & 8019 & 7996 \\
Tmin,Tmax & $0.836,0.879$ & $0.397,1.000$ \\
Tmin' & 0.483 &
\end{tabular}

Correction method $=$ \# Reported $\mathrm{T}$

Limits: Tmin $=0.397$

$\operatorname{Tmax}=1.000$

AbsCorr $=$ MULTI-SCAN

Data completeness $=0.997$

$\operatorname{Theta}(\max )=68.999$

$\mathrm{R}($ reflections $)=0.1272(4902)$

$\mathrm{wR} 2($ reflections $)=0.3858(7996)$

$\mathrm{S}=1.175 \quad \mathrm{Npar}=517$

Table S2. Crystal data and structure refinement for compound 2 


\section{Devices}

Commercially available interdigital Au electrodes $(15 \times 15 \mathrm{~mm} 2)$ from Fraunhofer IPM were used. High doped n-type silicon was used as gate electrode. A $30 \mathrm{~nm}$ Au electrode with a $10 \mathrm{~nm}$ high work function adhesion layer (ITO) (structured by lift-off technique) was patterned as source and drain electrodes respectively on the gate dielectric of 230-nm thermal-oxidized $\mathrm{SiO}_{2}$. First, the electrodes were cleaned one by one by ultrasonication with electronic grade acetone and isopropanol and dried under S3 compressed N2. Then, the electrodes were treated with oxygen plasma for 15 minutes. To form self-assembled monolayers (SAMs) of octadeciltrichlorosilane (OTs) on the $\mathrm{SiO}_{2}$ surface, the substrates were dipped into a solution of OTs $(4 \mathrm{mM})$ in toluene and heated at $50^{\circ} \mathrm{C}$ during 30 minutes. The modified electrodes were subsequently washed with chloroform and dried for 1 hour at $80^{\circ} \mathrm{C}$. Finally, the substrates were cleaned again with electronic grade acetone and isopropanol and dried with $\mathrm{N}_{2}$.

40-nm thick compounds $\mathbf{1}$ and $\mathbf{2}$ films were deposited by vacuum thermal deposition in an ultra high vacuum (UHV) evaporator (base pressure $<10^{-9} \mathrm{mbar}$ )) while the substrate temperatures were controlled at $25^{\circ} \mathrm{C}, 80^{\circ} \mathrm{C}$, and $120^{\circ} \mathrm{C}$ respectively. The electrical characterizations of the devices were measured with a Keithley 4200 semiconductor analyser system which connected to Lakeshore probe-station in vacuum at room temperature without light. The samples were exposed to air before being introduced in the vacuum probe station for a time smaller than 30 minutes. 
Table S3. Electrical characterization for compound 1 at different substrate temperatures (r.t, $80^{\circ} \mathrm{C}$ and $120^{\circ} \mathrm{C}$ ). Values of mobility, threshold voltage $(V \mathrm{t})$ and on/off ratio current (Ion/off) are reported.

\begin{tabular}{|c|c|c|c|c|c|c|c|c|c|c|}
\hline \multicolumn{2}{|c|}{ Compound 1} & \multicolumn{3}{|c|}{$25^{\circ} \mathrm{C}$} & \multicolumn{3}{|c|}{$80^{\circ} \mathrm{C}$} & \multicolumn{3}{|c|}{$120^{\circ} \mathrm{C}$} \\
\hline $\mathrm{W}(\mu \mathrm{m})$ & $\mathrm{L}(\mu \mathrm{m})$ & $\begin{array}{l}\text { Mobility } \\
\left(\mathrm{cm}^{2} / \mathrm{Vs}\right)\end{array}$ & $\begin{array}{c}V_{\mathrm{t}} \\
(\mathrm{V}) \\
\end{array}$ & $\mathrm{I}_{\mathrm{on} / \mathrm{fff}}$ & $\begin{array}{l}\text { Mobility } \\
\left(\mathrm{cm}^{2} / \mathrm{Vs}\right)\end{array}$ & $\begin{array}{c}V_{\mathrm{t}} \\
(\mathrm{V}) \\
\end{array}$ & $\mathrm{I}_{\mathrm{on} / \mathrm{off}}$ & $\begin{array}{l}\text { Mobility } \\
\left(\mathrm{cm}^{2} / \mathrm{Vs}\right)\end{array}$ & $\begin{array}{l}V \mathrm{t} \\
(\mathrm{V})\end{array}$ & $\mathrm{I}_{\text {on/off }}$ \\
\hline \multirow{4}{*}{10000} & \multirow{4}{*}{2,5} & $5,43 \mathrm{E}-05$ & $-17,50$ & 17,3 & $4,77 \mathrm{E}-07$ & $-8,5$ & 3,12 & $2,94 \mathrm{E}-08$ & 1,25 & 9,4 \\
\hline & & $1,18 \mathrm{E}-04$ & $-13,50$ & 28,7 & $3,94 \mathrm{E}-07$ & -9 & 2,94 & $4,44 \mathrm{E}-08$ & 1 & 11,9 \\
\hline & & $1,11 \mathrm{E}-04$ & $-15,98$ & 23,5 & $9,54 \mathrm{E}-08$ & $-15,5$ & 1,7 & & & \\
\hline & & $1,15 \mathrm{E}-04$ & $-18,50$ & 18,7 & $5,85 \mathrm{E}-07$ & $-14,7$ & 2,58 & & & \\
\hline \multirow{4}{*}{10000} & \multirow{4}{*}{5} & $1,05 \mathrm{E}-04$ & $-17,00$ & 18,7 & $3,52 \mathrm{E}-07$ & $-5,75$ & 5,2 & $5,10 \mathrm{E}-08$ & $-27,5$ & 26,2 \\
\hline & & $1,19 \mathrm{E}-04$ & $-21,00$ & 20,1 & $1,41 \mathrm{E}-07$ & $-6,5$ & 1,8 & & & \\
\hline & & $6,30 \mathrm{E}-05$ & $-16,50$ & 29 & $7,03 \mathrm{E}-08$ & $-3,75$ & 3,03 & $1,82 \mathrm{E}-08$ & $-17,5$ & 53,2 \\
\hline & & $9,82 \mathrm{E}-04$ & $-13,50$ & 19,2 & $5,98 \mathrm{E}-08$ & $-6,75$ & 1,44 & $2,73 \mathrm{E}-08$ & $-9,25$ & 5,99 \\
\hline \multirow{4}{*}{10000} & \multirow{4}{*}{10} & $1,35 \mathrm{E}-04$ & $-17,50$ & 16,8 & $2,49 \mathrm{E}-07$ & -10 & 12,2 & & & \\
\hline & & $9,06 \mathrm{E}-05$ & $-24,50$ & 11,5 & $7,55 \mathrm{E}-07$ & $-10,75$ & 5,5 & & & \\
\hline & & $1,42 \mathrm{E}-04$ & $-15,00$ & 17,6 & $4,01 \mathrm{E}-07$ & $-19,5$ & 10,75 & $1,29 \mathrm{E}-08$ & -28 & 12,3 \\
\hline & & $1,15 \mathrm{E}-04$ & $-13,50$ & 17,2 & $1,50 \mathrm{E}-06$ & -20 & 31,6 & $2,32 \mathrm{E}-08$ & -23 & 21,6 \\
\hline \multirow{4}{*}{10000} & \multirow{4}{*}{20} & $4,30 \mathrm{E}-04$ & $-24,00$ & 18 & $1,61 \mathrm{E}-06$ & $-14,5$ & 21 & & & \\
\hline & & $3,03 \mathrm{E}-04$ & $-19,50$ & 58,5 & $7,68 \mathrm{E}-07$ & $-13,5$ & 17,2 & & & \\
\hline & & $3,70 \mathrm{E}-04$ & $-19,50$ & 65,3 & $1,36 \mathrm{E}-06$ & $-17,5$ & 25,4 & & & \\
\hline & & $1,84 \mathrm{E}-04$ & $-14,00$ & 64,19 & $2,32 \mathrm{E}-06$ & $-17,5$ & 37 & & & \\
\hline & average: & $2,15 E-04$ & $-17,56$ & 27,8 & $6,96 \mathrm{E}-07$ & $-12,1$ & 11,4 & $2,95 \mathrm{E}-08$ & $-14,7$ & 20,1 \\
\hline
\end{tabular}

Table S4. Electrical characterization compound 2 at different substrate temperatures (r.t, $80^{\circ} \mathrm{C}$ and $120^{\circ} \mathrm{C}$ ). Values of mobility, threshold voltage $(V \mathrm{t})$ and on/off ratio current (Ion/off) are reported.

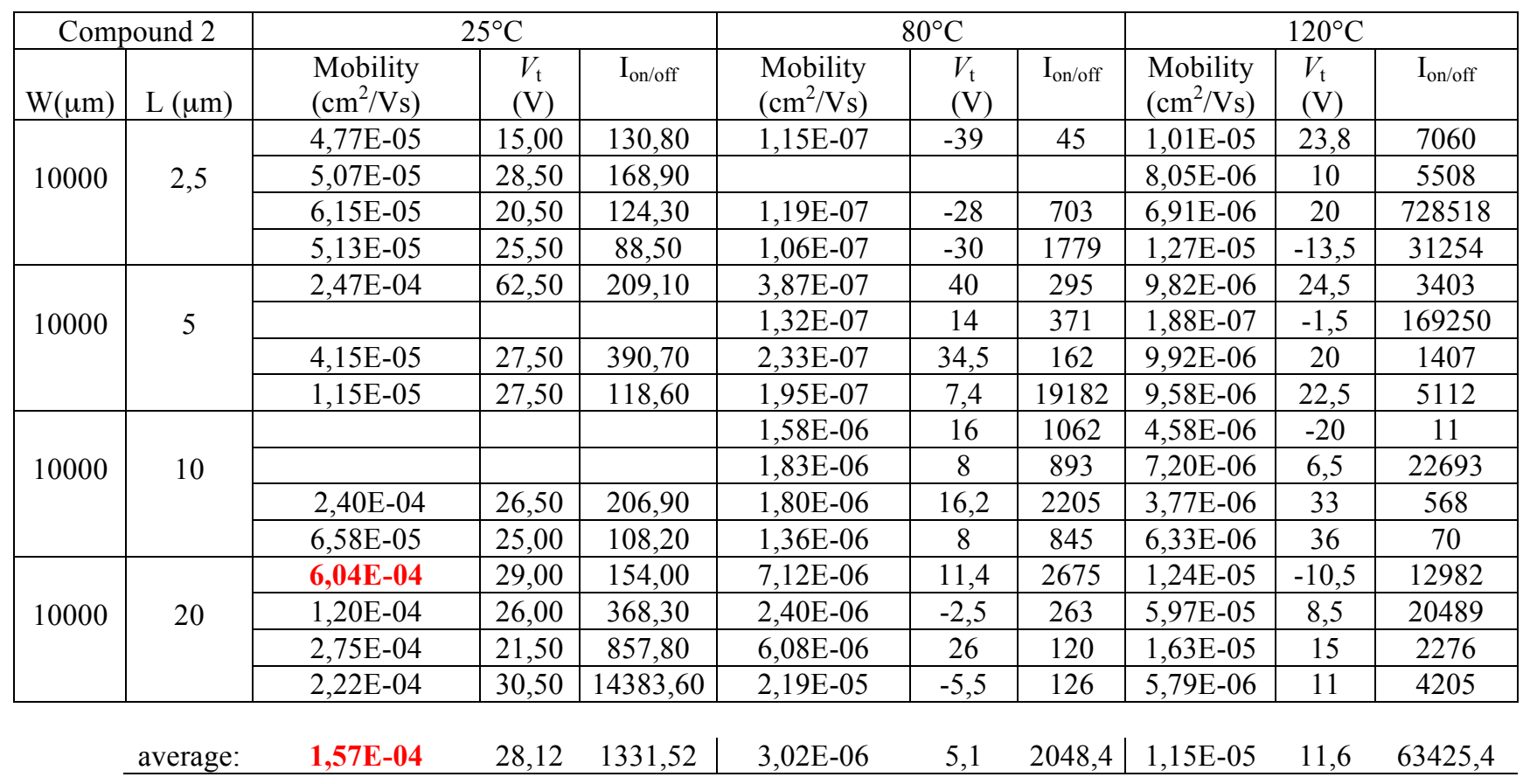




\section{AFM}

$5 \mu \mathrm{m} \times 5 \mu \mathrm{m} \quad \mathrm{RT}$
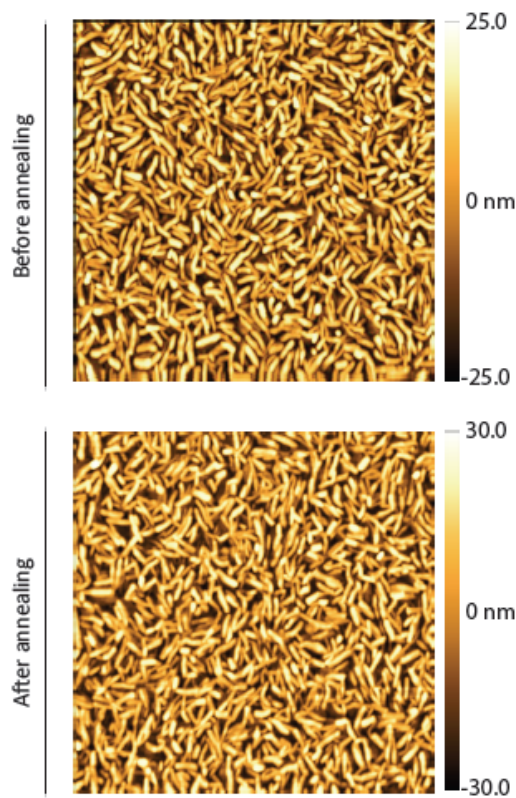

$80^{\circ} \mathrm{C}$
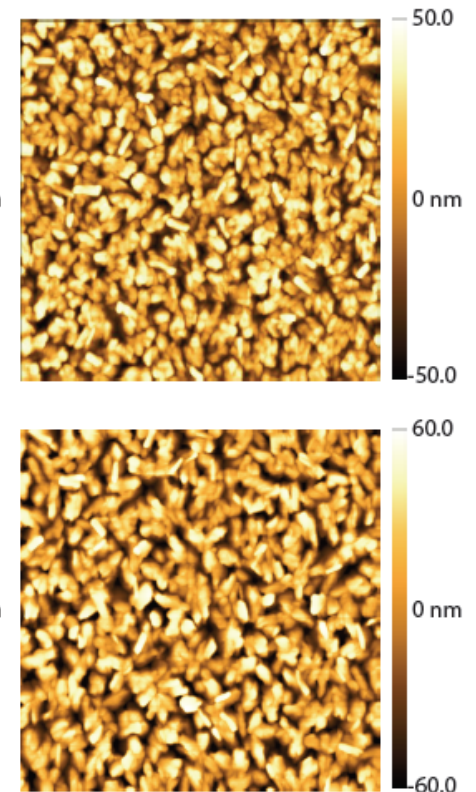

$120^{\circ} \mathrm{C}$
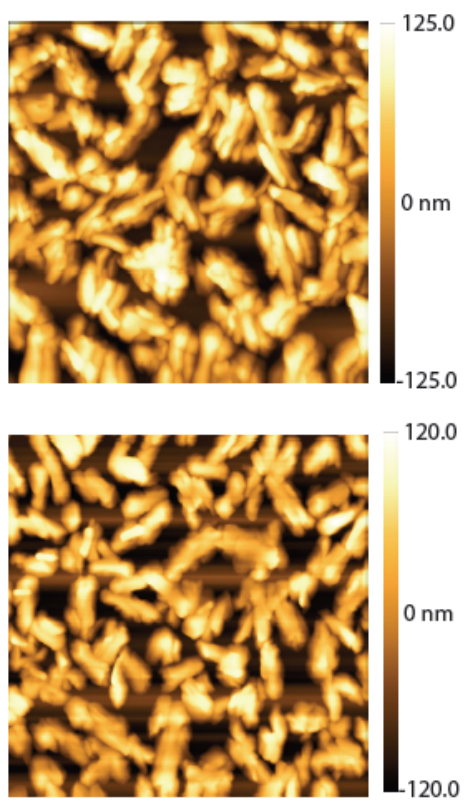

Figure S9 Representative thin film morphologies $(5 \mu \mathrm{m} \times 5 \mu \mathrm{m})$ of compound 1 thin films deposited at $25^{\circ} \mathrm{C}$ (left), $80{ }^{\circ} \mathrm{C}$ (centre) and $120^{\circ} \mathrm{C}$ (right) before (top) and after (bottom) annealing

RT
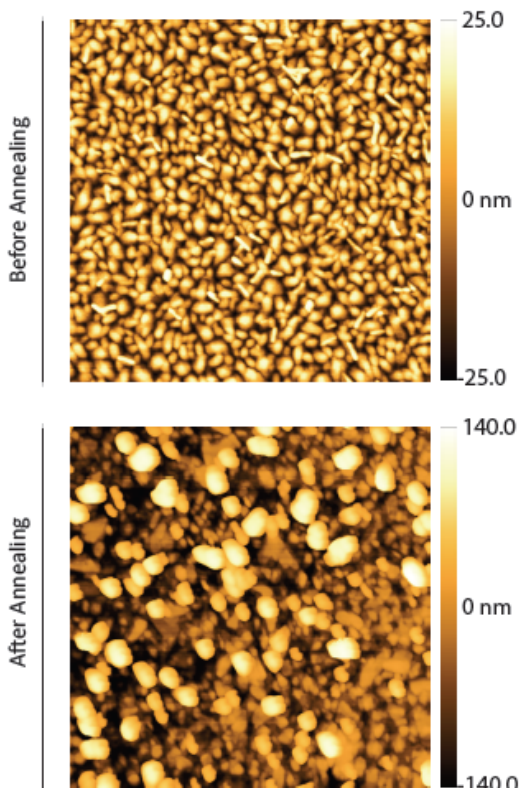

$80^{\circ} \mathrm{C}$
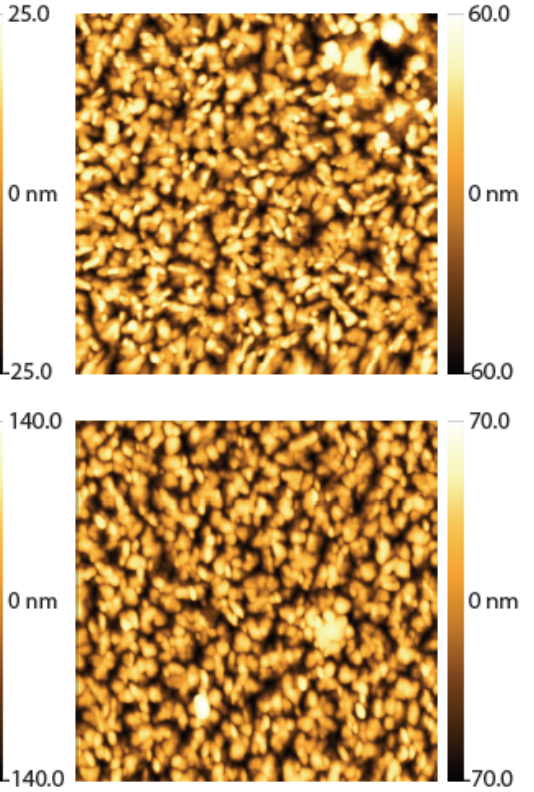

$120^{\circ} \mathrm{C}$
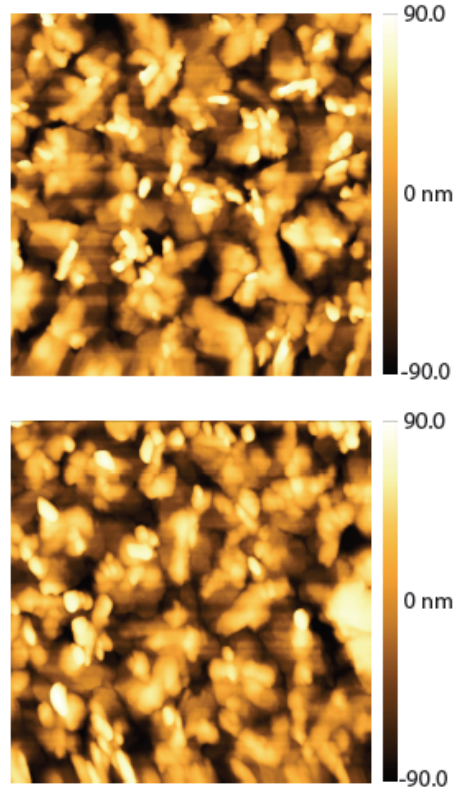

Figure S10 Representative thin film morphologies (5 $\mu \mathrm{m}$ x $5 \mu \mathrm{m})$ of compound 2 thin films deposited at $25^{\circ} \mathrm{C}$ (left), $80^{\circ} \mathrm{C}$ (centre) and $120^{\circ} \mathrm{C}$ (right) before (top) and after (bottom) annealing 


\section{Calculations}

\section{Frontier orbitals and energies}

Geometries were minimized at the B3LYP level with the 6-31g(d,p) basis set. All minima were checked by frequency calculations. TD-DFT, IP, EA, and frontier orbital energies were computed with the $6-311+\mathrm{g}(2 \mathrm{~d}, \mathrm{p})$ basis set with the B3LYP and BMK Hamiltonians. Calculations in vacuum and ODCB were performed and yielded similar results for the neutral molecules. Table S5 presents the frontier orbitals energies and the HOMO-LUMO gaps for B3LYP, and BMK in both gas phase and with an implicit solvent model of ODCB as well as the corresponding experimental values.

Table S5. Frontier orbitals computed with the B3LYP and BMK Hamiltonians with the 6$311+\mathrm{g}(2 \mathrm{~d}, \mathrm{p})$ basis set in odbc and vacuum from B3LYP-6-31g $(\mathrm{d}, \mathrm{p})$ geometries. All values in $\mathrm{eV}$.

\begin{tabular}{|l|l|l|l|l|l|}
\hline B3LYP/6-311+g(2d,p) & LUMO+1 & LUMO & HOMO & HOMO-1 & gap \\
\hline $0(0 \mathrm{~N})$ & -1.77 & -2.99 & -5.05 & -5.84 & 2.06 \\
\hline $1(2 \mathrm{~N})$ & -1.92 & -3.3 & -5.29 & -6.01 & 1.99 \\
\hline $2(4 \mathrm{~N})$ & -2.11 & -3.64 & -5.65 & -6.11 & 2.01 \\
\hline $0(0 \mathrm{~N})$ ODBC & -1.73 & -2.99 & -5.05 & -5.81 & 2.06 \\
\hline $1(2 \mathrm{~N})$ ODBC & -1.86 & $-\mathbf{3 . 3 1}$ & $-\mathbf{5 . 3 1}$ & -5.96 & $\mathbf{2 . 0 0}$ \\
\hline 2(4N)ODBC & -2.12 & $-\mathbf{3 . 6 6}$ & $-\mathbf{5 . 7 2}$ & -6.02 & $\mathbf{2 . 0 6}$ \\
\hline BMK/6-311+g(2d,p) & LUMO+1 & LUMO & HOMO & HOMO-1 & gap \\
\hline 0(0N) & -1.14 & -2.53 & -5.63 & -6.52 & 3.1 \\
\hline $1(2 \mathrm{~N})$ & -1.28 & -2.84 & -5.89 & -6.69 & 3.05 \\
\hline 2(4N) & -1.41 & -3.19 & -6.29 & -6.78 & 3.1 \\
\hline 0(0N) ODBC & -1.14 & -2.58 & -5.67 & -6.52 & 3.09 \\
\hline $1(2 \mathrm{~N})$ ODBC & -1.27 & -2.9 & -5.96 & -6.68 & 3.06 \\
\hline 2(4N)ODBC & -1.45 & -3.26 & -6.42 & -6.73 & 3.16 \\
\hline EXPERIMENTAL & & LUMO & HOMO & & gap \\
\hline $1(2 \mathrm{~N})$ ODBC & -3.57 & -5.43 & & 1.86 \\
\hline 2(4N)ODBC & -3.89 & & & & \\
\hline
\end{tabular}


Ionization Potentials (IP) and Electron Affinity (EA) values with the same Hamiltonians were computed and are presented in Table S6. In both cases the IP and EA values fit better the experimentally measured quantities than the HOMO and LUMO eigenvalues of Table S5, as it would be expected since for these values the oxidation (IP) and reduction (EA) of the molecules in solution are explicitly computed.

Table S6. Vertical ionization potentials (IP), electron affinities (EA) and gap (EA+IP) at the B3LYP and BMK 6-311+g(2d,p) levels in odbc from B3LYP-6-31g(d,p) geometries. All values in $\mathrm{eV}$.

\begin{tabular}{|l|l|l|l|}
\hline B3LYP/6-311+g(2d,p) & LUMO(EA) & HOMO(IP) & GAP (EA+IP) \\
\hline $0(0 \mathrm{~N})$ ODBC & -3.11 & 4.93 & 1.82 \\
\hline $1(2 \mathrm{~N})$ ODBC & -3.42 & 5.19 & 1.76 \\
\hline $2(4 \mathrm{~N})$ ODBC & -3.76 & 5.60 & 1.84 \\
\hline BMK/6-311+g(2d,p) & LUMO(EA) & HOMO(IP) & GAP (EA+IP) \\
\hline $0(0 \mathrm{~N})$ ODBC & -3.14 & 5.12 & 1.98 \\
\hline $1(2 \mathrm{~N})$ ODBC & $\mathbf{- 3 . 4 6}$ & $\mathbf{5 . 3 8}$ & $\mathbf{1 . 9 2}$ \\
\hline $2(4 \mathrm{~N})$ ODBC & $\mathbf{- 3 . 8 1}$ & $\mathbf{5 . 8 5}$ & $\mathbf{2 . 0 3}$ \\
\hline EXPERIMENTAL & LUMO & HOMO & GAP \\
\hline $1(2 \mathrm{~N})$ ODBC & $\mathbf{- 3 . 5 7}$ & $\mathbf{- 5 . 4 3}$ & $\mathbf{1 . 8 6}$ \\
\hline 2(4N)ODBC & $\mathbf{- 3 . 8 9}$ & & \\
\hline
\end{tabular}


In both frontier orbitals, HOMO and LUMO, the electronic density is mostly localized in the acetylene moiety and in the "tetracene" region. Interestingly, the pyrene moiety is consistently less populated and the $\mathrm{K}$ region has zero population in all cases. Remarkably all three molecules have very similar HOMO and LUMO orbitals which is consistent with the fact the HOMO and LUMO are $\pi$ molecular orbitals and the three molecules, 0,1 and 2, are isoelectronic. The computed gaps for the three molecules are similar (maximum difference is $0.1 \mathrm{eV}$ ), so the effect of substituting $>\mathrm{C}-\mathrm{H}$ by $>\mathrm{N}$ in these molecules stabilizes both the HOMO and LUMO by similar amounts.
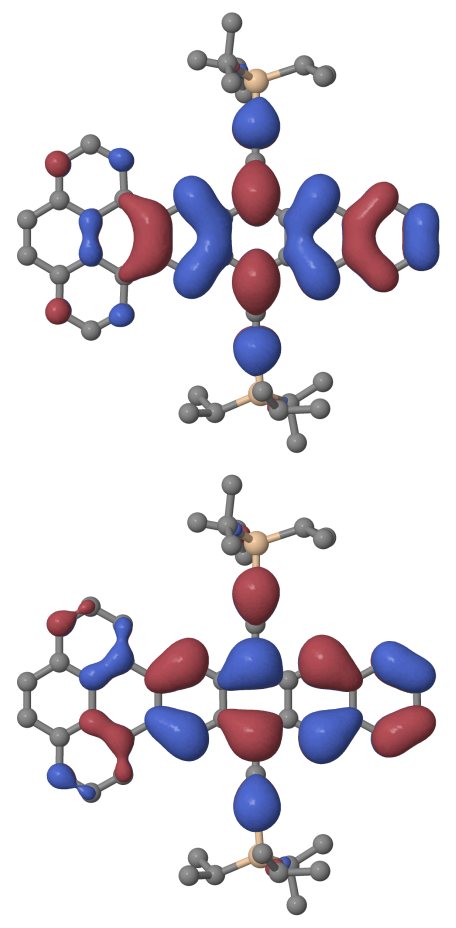

$0(0 \mathrm{~N})$
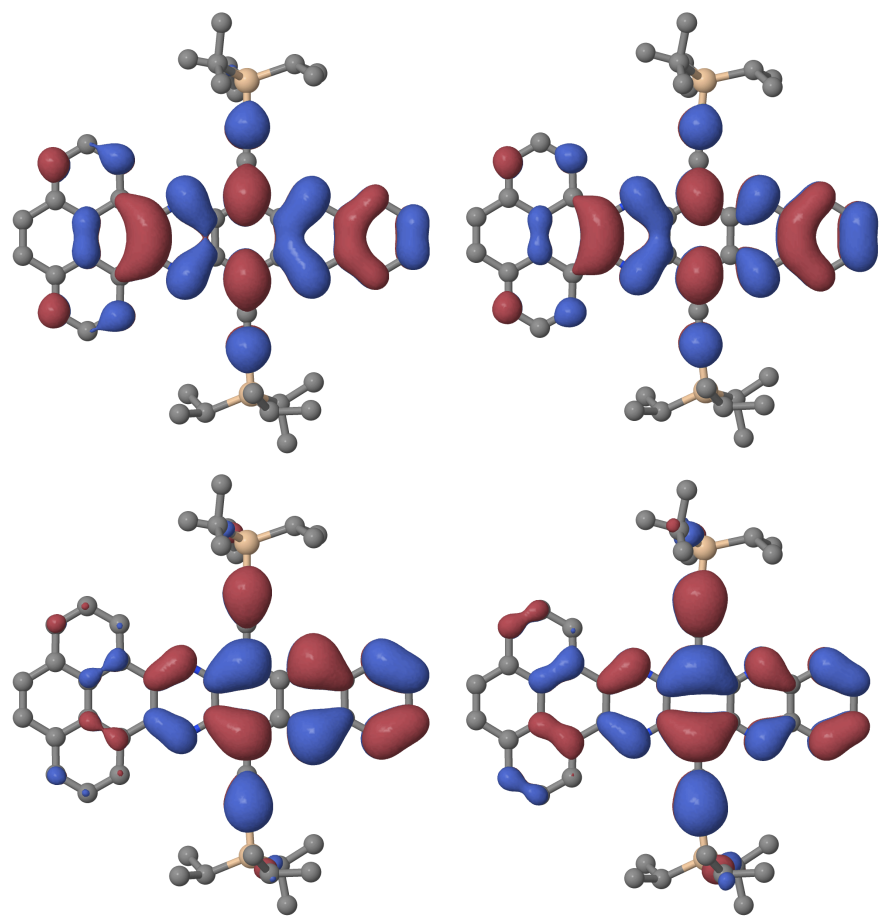

$1(2 \mathrm{~N})$

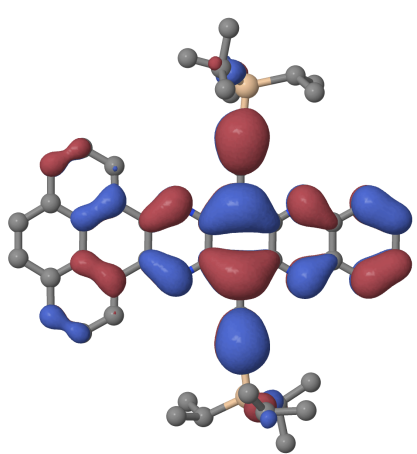

$2(4 \mathrm{~N})$

Figure S11. BMK-odbc-6311+g(2d,p)/B3LYP-odbc-6-31(d,p) level frontier orbitals: LUMO (top) and HOMO (bottom). 


\section{TD-DFT}

Table S7. BMK-odbc-6311+g(2d,p)/B3LYP-odbc-6-31(d,p) and B3LYP-odbc$6311+\mathrm{g}(2 \mathrm{~d}, \mathrm{p}) / \mathrm{B} 3 \mathrm{~L}$ YP-odbc-6-31(d,p), first two TD-DFT transitions and experimental values for the electronic spectra.

\begin{tabular}{|c|c|c|c|c|}
\hline BMK/6-311+g(2d,p) & $\mathrm{eV}$ & $\mathrm{nm}$ & Osc. Strength & Major contribs \\
\hline $0(0 \mathrm{~N}) \mathrm{ODBC}$ & 1.95 & 637 & 0.26 & HOMO->LUMO (100\%) \\
\hline $0(0 \mathrm{~N}) \mathrm{ODBC}$ & 2.85 & 435 & 0.39 & H-1->LUMO (89\%) \\
\hline $1(2 \mathrm{~N}) \mathrm{ODBC}$ & 1.89 & 657 & 0.20 & HOMO->LUMO (100\%) \\
\hline $1(2 \mathrm{~N}) \mathrm{ODBC}$ & 2.62 & 473 & 0.59 & H-1->LUMO (95\%) \\
\hline $2(4 \mathrm{~N}) \mathrm{ODBC}$ & 1.98 & 625 & 0.25 & HOMO->LUMO (100\%) \\
\hline $2(4 \mathrm{~N}) \mathrm{ODBC}$ & 2.41 & 515 & 0.63 & H-1->LUMO (96\%) \\
\hline \multicolumn{5}{|l|}{ B3LYP/6-311+g(2d,p) } \\
\hline $0(0 \mathrm{~N}) \mathrm{ODBC}$ & 1.74 & 714 & 0.20 & HOMO->LUMO (100\%) \\
\hline $0(0 \mathrm{~N}) \mathrm{ODBC}$ & 2.39 & 519 & 0.25 & H-1->LUMO (96\%) \\
\hline $1(2 \mathrm{~N}) \mathrm{ODBC}$ & 1.62 & 765 & 0.14 & HOMO->LUMO (100\%) \\
\hline $1(2 \mathrm{~N}) \mathrm{ODBC}$ & 2.21 & 561 & 0.37 & H-1->LUMO (98\%) \\
\hline $2(4 \mathrm{~N}) \mathrm{ODBC}$ & 1.69 & 733 & 0.18 & HOMO->LUMO (100\%) \\
\hline $2(4 \mathrm{~N}) \mathrm{ODBC}$ & 1.97 & 630 & 0.40 & H-1->LUMO (99\%) \\
\hline \multicolumn{5}{|l|}{ EXPERIMENTAL } \\
\hline $1(2 \mathrm{~N}) \mathrm{ODBC}$ & & 652 & & 1st transition \\
\hline $2(4 \mathrm{~N}) \mathrm{ODBC}$ & & 645 & & 1st transition \\
\hline
\end{tabular}




\section{References:}

[1] A. L. Appleton, S. M. Brombosz, S. Barlow, J. S. Sears, J.-L. Bredas, S. R. Marder and U. H. F. Bunz, Nat. Commun. 2010, 1, 91.

[2] B. D. Lindner, J. U. Engelhart, O. Tverskoy, A. L. Appleton, F. Rominger, A. Peters, H.-J. Himmel and U. H. F. Bunz, Angew. Chem. Int. Ed. 2011, 50, 8588-8591.

[3] J. Hu, D. Zhang and F. W. Harris, J. Org. Chem. 2005, 70, 707-708. 